\title{
iPractice: Piloting the Effectiveness of a Tablet-Based Home Practice Program in Aphasia Treatment
}

\author{
Jacquie Kurland, Ph.D., CCC-SLP, ${ }^{1}$ Abigail R. Wilkins, M.A., ${ }^{1}$ and \\ Polly Stokes, M.S., CCC-SLP ${ }^{1}$
}

ABSTRACT

Learning Outcomes: As a result of this activity, the reader will be able to discuss appropriate candidate selection for tablet-based home practice programs and summarize some of the inherent obstacles in using tablet-based technology for aphasia rehabilitation.

${ }^{1}$ Department of Communication Disorders, University of Massachusetts Amherst, Amherst, Massachusetts.

Address for correspondence: Jacquie Kurland, Ph.D., CCC-SLP, Department of Communication Disorders, University of Massachusetts Amherst, 358 North Pleasant Street, Amherst, MA 01003-9296 (e-mail: jkurland@comdis.umass. edu).
iRehab: Incorporating iPads and Other Tablets in Aphasia Treatment; Guest Editor, Jacquie Kurland, Ph.D., CCC-SLP

Semin Speech Lang 2014;35:51-64. Copyright (C) 2014 by Thieme Medical Publishers, Inc., 333 Seventh Avenue, New York, NY 10001, USA. Tel: +1(212) 584-4662. DOI: http://dx.doi.org/10.1055/s-0033-1362991. ISSN 0734-0478. 
The field of clinical speech-language pathology is progressively moving toward incorporating technology into treatment, with electronic tablets such as the iPad (Apple Inc., Cupertino, CA) at the forefront. ${ }^{1}$ In the last decade, for example, affordable cost, ease of use, portability, and a certain "it" factor have encouraged practitioners, patients, and families to reach for the $\mathrm{iPad}$, even if they were novices with such technology. New, customizable, aphasia-specific application software (apps) are becoming available in the iPad App Store regularly. A search for "aphasia" from within the iPad Apps returns many reputable companies that are not newcomers to aphasia therapy (e.g., Lingraphica (Princeton, NJ); Constant Therapy (Boston, MA); Tactus Therapy Solutions, Ltd (Vancouver, BC)). Perhaps most surprisingly, many of the apps are free.

Recently, several interested organizations (e.g., National Stroke Association) have published on their Web sites or in their monthly magazines (e.g., The ASHA Leader) lists of "aphasia apps." Some were not necessarily developed with aphasia treatment in mind and include apps that enable augmentative and alternative technology as well as those that may be used in delivering speech and language treatment. In the case of the latter, the criteria for judging the merit or effectiveness of the apps being endorsed is not always clear, although at times it is obvious that one or more authors may have, at least in part, a financial interest in the widespread use of particular apps. Last year, in response to the sudden ubiquity of apps available to clinicians, Holland and colleagues shared their experience in using apps in aphasia treatment programs and provided some guidelines for selecting/ judging appropriate apps and achieving buyin from clients. ${ }^{2}$ Although the effectiveness of the majority of aphasia-focused apps has yet to be vetted in the traditional manner of submitting methods and results of experimental inquiry for peer review, phase I clinical trials examining the efficacy of one or more aphasia apps are beginning to emerge. ${ }^{3}$ This new era of aphasia therapy has the potential to be groundbreaking for individuals with aphasia and the speech-language pathologists (SLPs) who treat them, as the technology offers unparalleled opportunities for massed, personalized practice in a portable package. Unfortunately, much like the excitement surrounding computer-assisted aphasia therapy a quarter century ago, the cart full of "aphasia treatment" goodies is already way ahead of the horse.

To our knowledge, there is very little research literature on the use of the iPad (or other tablet devices) in aphasia rehabilitation; however, this is likely to, and ought to, change in the next few years. Recently, Brandenburg and colleagues provided an integrated review of the literature pertaining to the accessibility and use of mobile technology by individuals with aphasia. ${ }^{4}$ Not surprisingly, the authors temper their excitement for this promising new approach with a call for more research evidence evaluating mobile technology's current and potential impact on the lives of people with aphasia.

Although the current literature is playing catch-up with the use of tablet technology in the clinic, several studies have investigated both the effectiveness of computer programs in aphasia therapy, as well as the effectiveness of home programs. ${ }^{5,6}$ Pederson and colleagues examined unsupervised computer rehabilitation of anomia. ${ }^{7}$ Using a program that targeted semantic, phonological, and written cueing, the authors found that, although performance varied, all three participants were able to improve their confrontation naming after computer treatment. More recently, Palmer et al conducted a randomized controlled trial of computer therapy for people with chronic aphasia. ${ }^{8}$ After 5 months of computer treatment, individuals in the experimental group were found to have significantly improved on confrontation-naming tasks in comparison with the control group.

Computerized home practice (HP) allows for individualized treatment, accessibility for those in rural and remote locations, and, perhaps most importantly, massed practice. Additionally, it allows for some autonomy, which may be lacking in traditional treatment programs. The portability of tablet devices combined with the technology to stream highquality audio, video, and other graphic stimulation, can enable individuals with aphasia to practice speech and language tasks literally whenever and wherever they choose. 
The current study aimed to investigate the effectiveness of an iPad-based HP program developed primarily by the first and second authors for maintaining and improving recent language treatment gains achieved by eight stroke survivors with chronic aphasia. The primary goals of the HP program were maintenance of treated words and new learning of untreated words, using unsupervised practice on individualized iPad practice programs. Our final discussion includes issues surrounding selection and training of appropriate candidates for tablet-based treatment, as well as the pros and cons of using this type of HP program.

\section{METHODS}

\section{Participants}

Eight right-handed, native English-speaking adults (four female), ages 55 to 81 (mean $=67.38$; standard deviation $=8.99$ ), were at least 8 months post-single unilateral cerebrovascular accident affecting the language dominant hemisphere at the time of baseline testing. Participant demographics can be seen in Table 1, including aphasia classification and severity. All subjects participated in an intensive 2-week aphasia treatment program prior to beginning the individualized HP programs. Three of the eight subjects are not included in the present data analysis. Two participants failed to complete the home program; one completed the program but his iPad was inadvertently programmed with the wrong practice stimuli.

\section{Materials}

Treatment stimuli consisted of black-and-white line drawings of common objects and picturable actions. ${ }^{9,10}$ Immediately following intensive language action treatment, individualized interactive books were created for each participant using iBooks Author software (Apple Inc., Cupertino, CA) and were distributed on iPads. Two books were created for each participant: one contained objects and one contained actions. Each book contained 20 words to be practiced; 10 had been used in the intensive 2 -week treatment program ("trained") and 10 were from a matched list of words not used in the treatment program ("untrained"). Both trained and matched untrained word lists were originally derived from words that each participant could not name in three of three baseline attempts. Following treatment, to-be-practiced lists were again matched on variables including word frequency, length, and visual complexity, in addition to accuracy on posttreatment probes.

Each word to be practiced had a chapter that contained five interactive pages targeting various levels of semantic, phonological, and orthographic cueing. The first interactive page of each chapter included a copy of the line drawing, a video (headshot) of a student slowly enunciating the word, and a series of tasks to scroll through that offered different kinds and levels of cueing. For example, in the chapter for football, the participant would scroll and see the following: (1) "What is it?"; (2) a series of eight hyphens, one for each letter of the word; (3) "The quarterback threw the - - - - - - -"; (4) a series of eight dashes including the initial letter $f$, and (5) a series of eight dashes spelling out the word, $-f-o-o-t-b-a-l-l$. Both the video and the scrolling tasks could be enlarged to full screen. The video could be played over and over and participants were encouraged to do so and then to repeat the word until they felt confident in their own production of the word. The second page was a word/picture-matching task, for example, "Which of these is a football?" wherein foils included a baseball, a basketball, and a soccer ball. The participant's task was to click on the correct response. The answer could be checked for accuracy and participants were encouraged to do so. Page 3 varied depending on whether the word had a natural rhyme. For example, for the word cherry, the instruction was "Choose the word that rhymes with the picture" and the choices were cheesy, berry, itchy, and sorry. In the case of football, with no natural rhyme, there was instead a picture/word matching task that included a copy of the line drawing along with the instruction "Choose the word that is spelled correctly" and four choices: football, futbowl, fotboll, footbell. In either case, the correct answer could be checked. Page 4 asked, "What doesn't belong?" and included four choices, in this case: touchdown, tackle, throw, and bat. Again, the answer could be 
Table 1 Participant Demographics and BDAE Selected Subtest Scores

\begin{tabular}{|c|c|c|c|c|c|c|c|c|}
\hline & \multicolumn{8}{|c|}{ Participant Code } \\
\hline & $\overline{\mathrm{ACL}}$ & MCR & NWS & PBS & SSM & TJN & VKR & QDN \\
\hline Aphasia classification & Wernicke & Anomia & Anomia & $\begin{array}{l}\text { Transcortical } \\
\text { sensory }\end{array}$ & Anomia & $\begin{array}{l}\text { Mixed } \\
\text { transco- } \\
\text { rtical }\end{array}$ & $\begin{array}{l}\text { Crossed } \\
\text { Wernicke }\end{array}$ & Conduction \\
\hline $\begin{array}{l}\text { Aphasia severity rating scale } \\
(\max 5)\end{array}$ & 2 & 1.5 & 2.5 & 2 & 4 & 1.5 & 2 & 2 \\
\hline Gender & M & $\mathrm{F}$ & $\mathrm{F}$ & $\mathrm{F}$ & M & M & M & $\mathrm{F}$ \\
\hline Age when tested at baseline & 58 & 70 & 67 & 80 & 63 & 55 & 63 & 78 \\
\hline $\begin{array}{l}\text { Time postonset (mo) } \\
\text { at baseline } \\
\text { I. Auditory comp (mean of } \\
3 \text { percentiles) }\end{array}$ & 84 & 70 & 24 & 17 & 25 & 19 & 8 & 8 \\
\hline Pre-TX & 18th & $82 n d$ & 72nd & $33 r d$ & $83 r d$ & 25th & $31 s t$ & $62 n d$ \\
\hline Post-HP & $43 r d$ & 80th & 78th & 50th & 67 th & 27 th & $\mathrm{n} / \mathrm{a}$ & $\mathrm{n} / \mathrm{a}$ \\
\hline a. Word discrimination (max & $37)$ & & & & & & & \\
\hline Pre-TX & 29.5 & 36.5 & 36.5 & 32 & 36 & 34 & 30.5 & 33 \\
\hline Post-HP & 33.5 & 37 & 36.5 & 35 & 35 & 33.5 & $\mathrm{n} / \mathrm{a}$ & $\mathrm{n} / \mathrm{a}$ \\
\hline b. Commands (max 15) & & & & & & & & \\
\hline Pre-TX & 10 & 15 & 13 & 10 & 15 & 6 & 9 & 14 \\
\hline Post-HP & 13 & 14 & 14 & 14 & 14 & 7 & $\mathrm{n} / \mathrm{a}$ & $\mathrm{n} / \mathrm{a}$ \\
\hline c. Complex ideational (max & 12) & & & & & & & \\
\hline Pre-TX & 3 & 9 & 10 & 7 & 10 & 4 & 8 & 10 \\
\hline Post-HP & 6 & 10 & 11 & 6 & 10 & 5 & $\mathrm{n} / \mathrm{a}$ & $\mathrm{n} / \mathrm{a}$ \\
\hline $\begin{array}{l}\text { II. Repetition } \\
\text { a. Single words ( } \max 10)\end{array}$ & & & & & & & & \\
\hline Pre-TX & 6 & 10 & 8 & 6 & 10 & 9 & 8 & 7 \\
\hline Post-HP & 7 & 10 & 10 & 8 & 10 & 9 & $\mathrm{n} / \mathrm{a}$ & $\mathrm{n} / \mathrm{a}$ \\
\hline b. Sentences (max 10) & & & & & & & & \\
\hline Pre-TX & 0 & 7 & 7 & 4 & 9 & 3 & 2 & 2 \\
\hline Post-HP & 2 & 9 & 9 & 4 & 10 & 4 & $\mathrm{n} / \mathrm{a}$ & $\mathrm{n} / \mathrm{a}$ \\
\hline III. Naming & & & & & & & & \\
\hline a. Visual confrontation (BNT & ; $\max 60)$ & & & & & & & \\
\hline Pre-TX & 23 & 37 & 47 & 43 & 48 & 12 & 41 & 42 \\
\hline Post-HP & 23 & 35 & 54 & 32 & 43 & 19 & $\mathrm{n} / \mathrm{a}$ & $\mathrm{n} / \mathrm{a}$ \\
\hline b. Responsive naming (max & 20) & & & & & & & \\
\hline Pre-TX & 4 & 12 & 15 & 15 & 20 & 6 & 13 & 18 \\
\hline Post-HP & 6 & 20 & 20 & 16 & 20 & 8 & $\mathrm{n} / \mathrm{a}$ & $\mathrm{n} / \mathrm{a}$ \\
\hline c. Naming actions $(\max 12)$ & & & & & & & & \\
\hline Pre-TX & 6 & 6 & 9 & 7 & 10 & 3 & 9 & 9 \\
\hline Post-HP & 7 & 11 & 12 & 9 & 10 & 4 & $\mathrm{n} / \mathrm{a}$ & $\mathrm{n} / \mathrm{a}$ \\
\hline d. Naming animals ( $\max 12$ & & & & & & & & \\
\hline Pre-TX & 6 & 10 & 11 & 10 & 10 & 12 & 10 & 7 \\
\hline Post-HP & 11 & 8 & 12 & 8 & 10 & 10 & $\mathrm{n} / \mathrm{a}$ & $\mathrm{n} / \mathrm{a}$ \\
\hline e. Naming tools (max 12 ) & & & & & & & & \\
\hline Pre-TX & 5 & 4 & 8 & 5 & 9 & 3 & 8 & 7 \\
\hline Post-HP & 5 & 5 & 9 & 6 & 10 & 6 & $\mathrm{n} / \mathrm{a}$ & $\mathrm{n} / \mathrm{a}$ \\
\hline
\end{tabular}

Abbreviations: BDAE, Boston Diagnostic Aphasia Examination; BNT, Boston Naming Test ${ }^{16}$; HP, home practice; max, maximum; $n / a$, not applicable; Tx, treatment. 
checked. A final page included a copy of the line drawing and the word and a place to check that the chapter had been completed.

Action books had a similar organization, with slightly different content. In lieu of the question "What is it?" on page 1, the question was "What's happening?"; the page was otherwise identical (i.e., the line drawing, the video, and the series of scrolling cues). Page 2 in the action books contained the word at the top and a video of the action being performed. The videos were $\sim 5$ to 8 seconds long and ended with a voiceover of the name of the action. Pages 3 to 4 were word/picture and picture/ word matching tasks, and page 5 asked, "Which picture does NOT match the word [e.g., STIR]?" with a series of four-color images that implied action using the target in different contexts. The target for this "odd-man-out" task was generally semantically related. For example, in the chapter "STIR," the correct answer depicted a spatula scraping remains of batter from a bowl. Action words were always printed and spoken in the infinitive.

Targets and foils utilized in the various tasks were high-quality color photographs downloaded from Internet sites such as Creative Commons, Flickr, and Google Images. They were scaled to proportionally fit the iBooks pages in Adobe Photoshop (Adobe Systems, Inc., San Jose, CA). Lexical stimuli for the "What is it?" and "What's happening?" routines were produced in Paint (Microsoft Corp., Redmond, WA). Videos were predominantly homemade utilizing an iPad, with clips edited as necessary in iMovie (Apple Inc., Cupertino, CA). In all cases, visual simplicity was prioritized.

\section{Procedures}

After a 2-week intensive treatment program in which participants were randomly assigned to receive either Intensive Language Action Therapy $^{11,12}$ or a modified version of Promoting Aphasic Communicative Effectiveness, ${ }^{13,14}$ and following posttreatment testing, the participants were introduced to the $\mathrm{iPad} 3$ loaded with their unique iBooks practice programs. Each iPad contained individualized sets of trained-tobe-practiced (TR-PR) and untrained-to-bepracticed (UNTR-PR) words in two books, Objects and Actions. Only one of the eight participants had any prior experience using an iPad or other tablet device and half of them had little to no prior experience with either a $\mathrm{PC}$ or Mac computer. Therefore, participants received variable degrees of training on how to navigate the iPad, depending on their comfort level (Table 2). One participant (no. 3) owned her own iPad2 and her iBooks were installed on her iPad. In all other cases, the layout of the home

Table 2 Participant Prior Experience and Required Training

\begin{tabular}{lllll}
\hline Participant No. & $\begin{array}{l}\text { Prior Experience } \\
\text { with iPad or } \\
\text { Other Tablet }\end{array}$ & $\begin{array}{l}\text { Prior Experience } \\
\text { with PC or Mac }\end{array}$ & $\begin{array}{l}\text { Training Required } \\
\text { for Basic iPad } \\
\text { Proficiency (h) }\end{array}$ & $\begin{array}{l}\text { Training Required } \\
\text { for Basic GoToMeeting } \\
\text { Proficiency (h) }\end{array}$ \\
\hline 1 & No & Low & 2.0 & 2.25 \\
2 & No & Low & 4.0 & 1.5 \\
3 & Yes ${ }^{*}$ & High & 1.0 & 0.5 \\
4 & No & None & 5.5 & 2.25 \\
5 & No & High & 0.5 & 0.3 \\
6 & No & High & 1.5 & 2.5 \\
7 & No & High & 0.75 & $\$$ \\
8 & No & None & $5.0^{\dagger}$ & $\neq$ \\
Mean & & 2.53 & 1.54 \\
Standard deviation & & & 2.00 & 0.97
\end{tabular}

*Patient no.3 owned an iPad2 (Apple Inc., Cupertino, CA) and her iBooks (Apple Inc., Cupertino, CA) were installed on her personal iPad.

${ }^{\dagger}$ Patient no. 8 had $5 \mathrm{~h}$ of training in the clinic and home, but never reached independent proficiency.

${ }^{\ddagger}$ Patient no. 8 practiced at home sporadically for $\sim 3$ wk and never received GoToMeeting (Citrix Online, Goleta, CA) training.

${ }^{\S}$ Patient no. 7 had personal issues that prevented him from participating in the home practice program. 
screen was changed so that only the apps required for HP (iBooks [Apple Inc., Cupertino, CA], GoToMeeting [Citrix Online, Goleta, CA] and Clock [Meanterm, Inc., San Francisco, CA]) were immediately available. This was done to minimize distraction.

Initial training included a very basic introduction to the device, how to power it on/off, how to find the home screen, how to charge it, how to place it in a stand, how to scroll, and so on. Several participants were intermittently unsuccessful scrolling with their fingers and were trained to use a stylus. Once a participant was comfortable with iPad basics, they were introduced to the iBooks app and trained to move through the chapters, switch books in the library, as well to practice their word sets. They were encouraged to maximize the number of trials and amount of practice and to practice saying and repeating their words out loud, but also to minimize incorrect productions by returning to the video model of the correct production whenever they were uncertain. They were asked to try and practice for at least 20 minutes, 5 or 6 days per week.

Participants were also trained to locate, start, and stop a digital stopwatch on the $\mathrm{iPad}$ home screen before and after practice to track daily usage. After each self-initiated practice session, the subjects would copy into a $\log$ the numbers displayed on the stopwatch.

Once the SLP (P.S.) was confident that the participants could practice independently at home, they signed a contract agreeing to care for the device and bring it back after 6 months. The SLP then delivered an iPad, carrying case, charger, stylus, and extension cord to each of seven participants who did not own one. The home visit was important because it allowed the SLP to train the participant along with anyone who might be available in the home (spouse, child, etc.) in how to troubleshoot potential problems with power, audio signal, connectivity, and so on. Moreover, it was essential to ensure that the iPad would pick up a $\mathrm{Wi}-\mathrm{Fi}$ signal in homes that already had wireless service or to determine if the participant would need monthly cellular service on the iPad device. In the latter case, it was provided free of charge. The home visit also allowed the SLP to begin the on-site training in how to connect weekly with her via the GoToMeeting app.
Participants met with the SLP on their iPad, using GoToMeeting, once a week at a regular prescheduled time. Telepractice sessions predominantly consisted of informal conversation but included a series of clinicianinitiated questions, such as if the participant had been practicing using the $\mathrm{iPad}$, if they were remembering to track how much time they spent practicing, if they were having any problems, and feedback regarding the participant's comfort and confidence in using the device. Once a month, for 6 months, participants returned to the clinic for probe testing to track progress on trained and untrained, practiced and unpracticed words.

\section{RESULTS}

Of the eight participants completing the initial intensive treatment protocol and training for the HP program, only six completed the program. One (no. 8) was disinterested in the iPad and $\mathrm{HP}$ and returned it within a month; one (no. 7) was undergoing personal and financial crises and returned the iPad in a nonworking condition within a few weeks. One participant (no. 6) practiced faithfully; unfortunately however, due to a technical error that went unnoticed for the first 2 months, he was practicing two sets of UNTR-PR objects, rather than half trained and half untrained. Results for the remaining five participants are reported here.

Participants reported practicing, on average, $\sim 18$ minutes per day or approximately 2 hours per week (Table 3). Six participants checked in with the SLP every week for 6 months, except for a 2-week break over the holidays in December. Over the course of the first few weeks, they experienced less and less trouble connecting on GoToMeeting and reported increasing ease and proficiency using the iBooks. On occasion, the SLP would be called to troubleshoot one issue or another with the iPad. Common problems included participants inadvertently turning down or turning off the volume or forgetting how to get back to the library. Some of the participants, particularly those with hemiparesis, occasionally had difficulty getting the book index to appear. On one occasion, one participant accidentally deleted both of the iBooks and they had to be reinstalled. 
Table 3 Average Time Participants Spent Practicing on iPads (Apple Inc., Cupertino, CA)

\begin{tabular}{llll}
\hline Participant No. & Daily Average (h:min:s) & Weekly Average (h:min:s) & Monthly Average (h:min:s) \\
\hline 1 & $0: 21: 10$ & $2: 16: 45$ & $11: 10: 40$ \\
2 & $0: 09: 52$ & $1: 07: 55$ & $4: 23: 29$ \\
3 & $0: 19: 43$ & $2: 11: 46$ & $9: 14: 14$ \\
4 & $0: 19: 42$ & $2: 23: 27$ & $9: 10: 51$ \\
5 & $0: 18: 31$ & $2: 05: 18$ & $8: 12: 30$ \\
Mean & $0: 17: 48$ & $2: 01: 02$ & $8: 26: 21$ \\
Standard deviation & $0: 04: 32$ & $0: 30: 26$ & $2: 30: 22$ \\
\hline
\end{tabular}

Progress was tracked monthly for words in four training and practice conditions: trained words that were practiced (TR-PR) in the HP program, untrained words that were practiced in the HP program (UNTR-PR), trained words that were not practiced (TR-UNPR), and untrained words that were not practiced (UNTRUNPR). All five participants in the HP study reported here showed a trend toward maintenance or continued improvement of the TR-PR words, and most (four of five) demonstrated dramatic gains in UNTR-PR words. These results contrast with the UNTR-UNPR condition, which remained relatively unchanged for all participants throughout the course of treatment and beyond (Figs. 1 to 5). Results of the daily treatment probes and posttreatment probes are included in the figures to demonstrate the role of practice on treatment outcomes.

All five participants demonstrated what Ramsberger and Marie suggest is strong evi- dence of a treatment effect during $\mathrm{HP}$ on UNTR-PR words with variable effect sizes on TR-PR words (Table 4). ${ }^{6}$ Visual inspection of the graphs reveals increases in mean level of performance, particularly for UNTR-PR words, which had no overlap with baseline (posttreatment) data points and an increase in the baseline slope. Clinical significance, based on at least $20 \%$ change in performance, was achieved on all practiced words.

In addition to naming on probes (the primary outcomes measure), participants were retested on standardized measures of aphasia after 6 months of HP. With few exceptions, their post-HP scores were equal to or better than their pretreatment scores on subtests of naming, auditory comprehension, and repetition from the Boston Diagnostic Aphasia Examination and the Boston Naming Test (Table 1). ${ }^{15,16}$ Analysis of changes in discourse post-HP is ongoing.

\section{ACL: Effect of Training and Practice on Naming Performance}

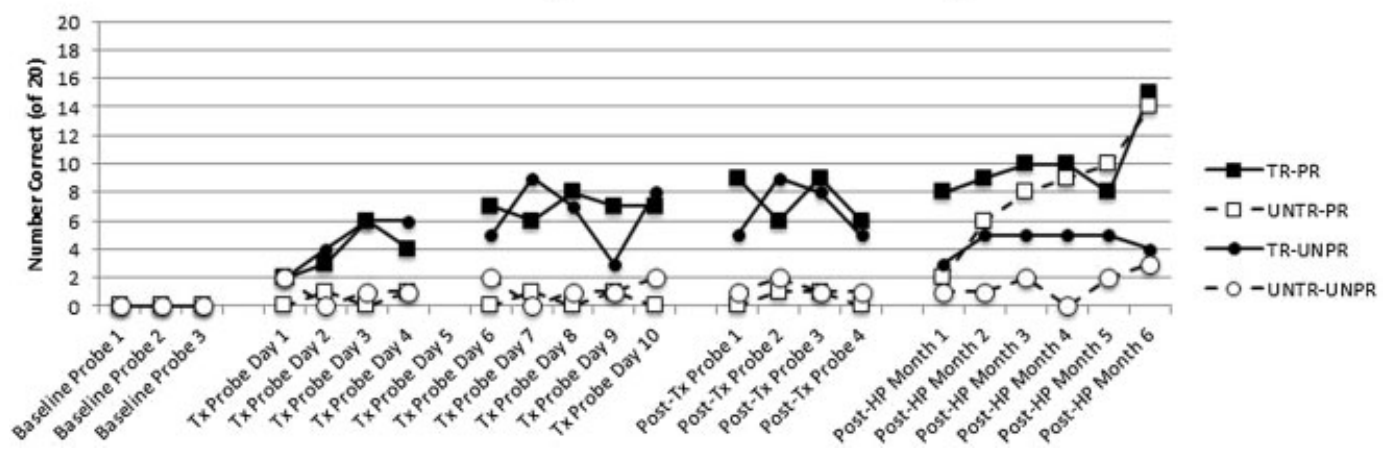

Figure 1 Patient 1: effect of training and practice on naming performance. Patient 1's picture-naming accuracy during probes for TR, UNTR, PR, and UNPR targets. Each set contained 10 picturable actions and 10 common objects. Abbreviations: HP, home practice; PR, practiced; TR, trained; Tx, treatment; UNPR, unpracticed; UNTR, untrained. 


\section{MCR: Effect of Training and Practice on Naming Performance}

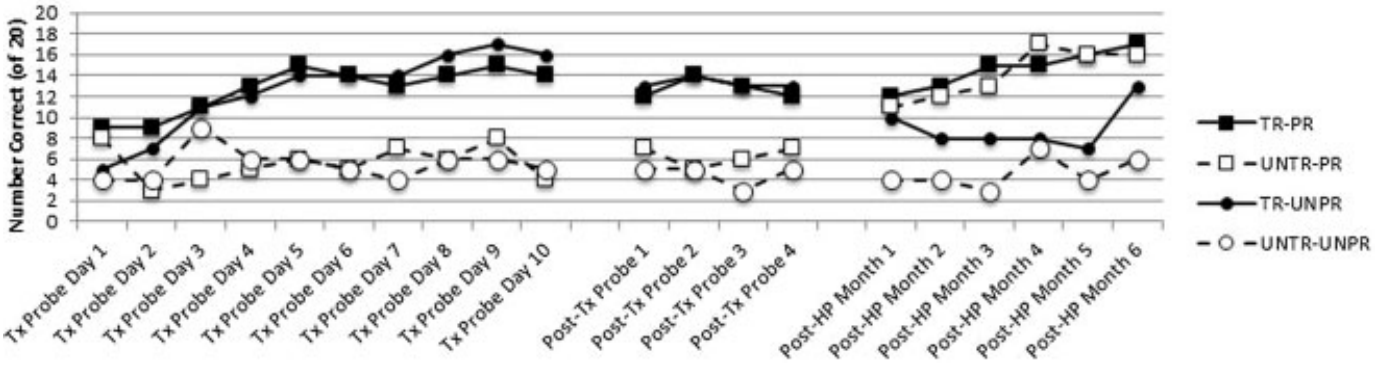

Figure 2 Patient 2: effect of training and practice on naming performance. Patient 2's picture-naming accuracy during probes for TR, UNTR, PR, and UNPR targets. Each set contained 10 picturable actions and 10 common objects. Abbreviations: HP, home practice; PR, practiced; TR, trained; Tx, treatment; UNPR, unpracticed; UNTR, untrained.

NWS: Effect of Training and Practice on Naming Performance

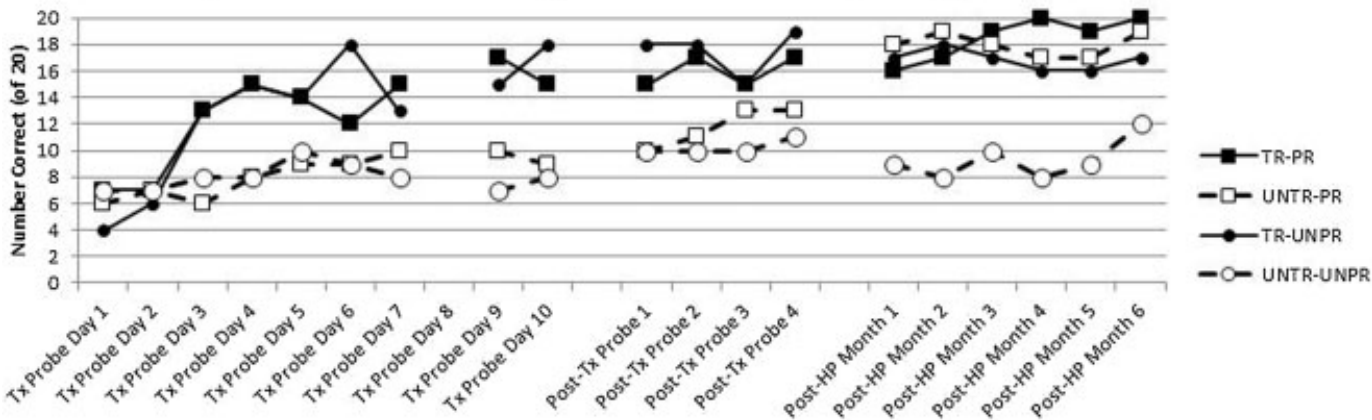

Figure 3 Patient 3: effect of training and practice on naming performance. Patient 3's picture-naming accuracy during probes for TR,UNTR, PR, and UNPR targets. Each set contained 10 picturable actions and 10 common objects. Abbreviations: HP, home practice; PR, practiced; TR, trained; Tx, treatment; UNPR, unpracticed; UNTR, untrained.

PBS: Effect of Training and Practice on Naming Performance

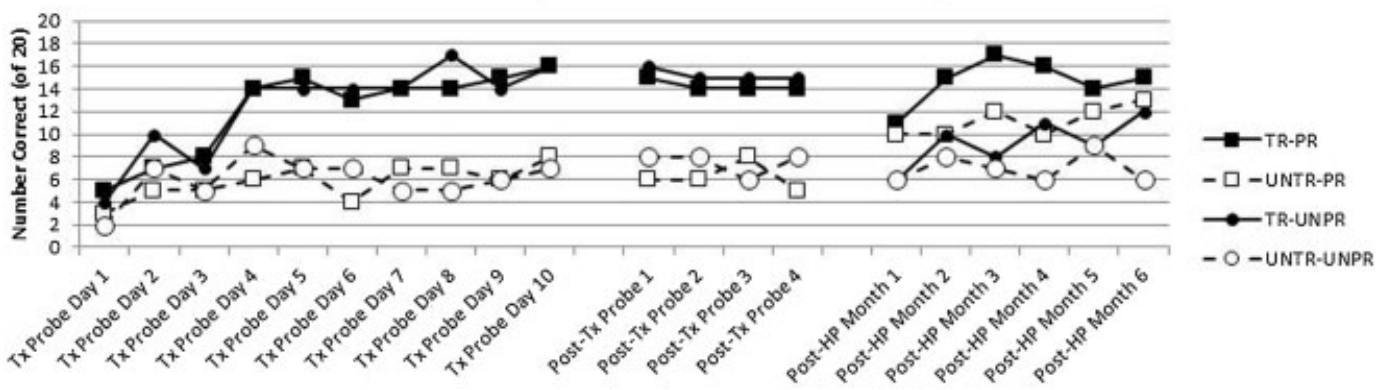

Figure 4 Patient 4: effect of training and practice on naming performance. Patient 4's picture-naming accuracy during probes for TR, UNTR, PR, and UNPR targets. Each set contained 10 picturable actions and 10 common objects. Abbreviations: HP, home practice; PR, practiced; TR, trained; Tx, treatment; UNPR, unpracticed; UNTR, untrained. 
SSM: Effect of Training and Practice on Naming Performance

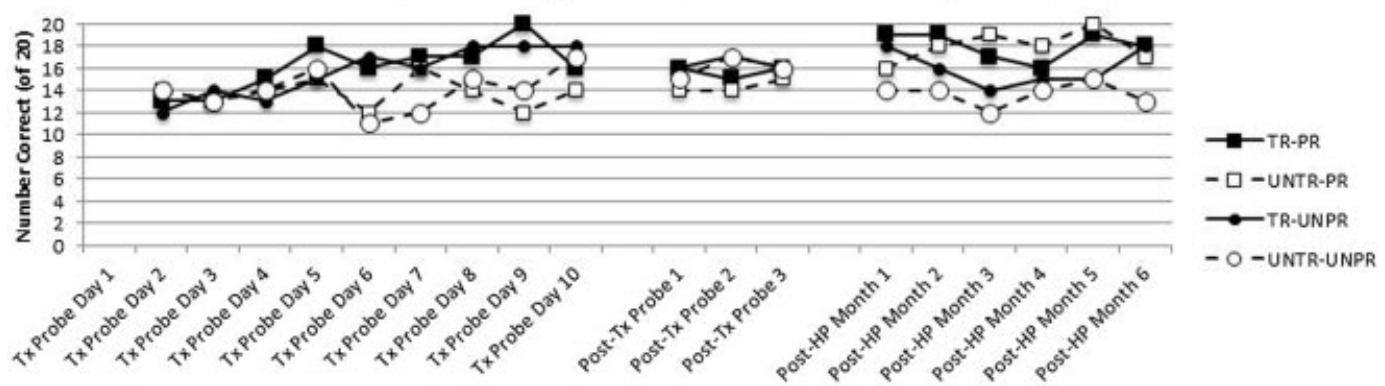

Figure 5 Patient 5: effect of training and practice on naming performance. Patient 5's picture-naming accuracy during probes for TR, UNTR, PR, and UNPR targets. Each set contained 10 picturable actions and 10 common objects. Abbreviations: HP, home practice; PR, practiced; TR, trained; Tx, treatment; UNPR, unpracticed; UNTR, untrained.

\section{DISCUSSION}

The results of this study demonstrate that HP on the iPad enabled maintenance and improvement over a period of 6 months of naming gains that were made during a 2 -week intensive language treatment program. It is anticipated that the participants who completed the HP program will complete an additional follow-up following a 6-month period of no practice.

In addition to maintaining or improving on gains achieved during treatment, participants were able to teach themselves words that were not previously trained. This evidence provides support for a cornerstone of neurorehabilitation: massed practice promotes coincidence (or Hebbian) learning. ${ }^{12}$ Most notably, our findings suggest that after making significant progress in short-term, intensive language therapy, people with chronic aphasia are able to maintain and improve autonomously upon these gains with a tablet-based HP program.

Although we report here on what appears to be a naming-oriented treatment approach, the assessment of treatment outcomes in this ongoing study includes tests that attempt to measure changes in discourse, functional outcomes and social participation, as well as evidence of changes in neurobiological mechanisms supporting improved outcomes. Reporting such data is beyond the scope of the current article; however, it is worth noting that participant responses to questions regarding the iPad-based HP program included comments such as: "I thought the practice was beneficial, um, I, I learned to speak well and, um, the, um, words came more freely"; "I think that's awesome"; "I love it, I just every day I do"; and "I'd turn it on then I can do 'em myself ... and then everyday try, try, try." Likewise, family members have reported, for example: "He uses longer sentences and is participating more in conversation with family and friends"; "His confidence is much higher"; and "The change in her is palpable since she started your study."

One of the most satisfying aspects of participants' progress was the degree to which they were surprised and delighted by their ability to learn to use the device and the GoToMeeting app. At least half of participants entered the program expressing some skepticism that they would become proficient with the iPad. The pleasure they expressed whenever they independently connected during the initial GoToMeeting telepractice sessions was priceless. At the same time, it is impossible to measure to what degree their success in the HP program was dependent on the cheerleading provided by those weekly sessions.

It is also noteworthy that the HP programs, and indeed the layout of the iBooks and the homepage, were designed specifically to accommodate a non-tech-savvy user. The initial indepth training on the $\mathrm{iPad}$ and practice program, on-site home training with the GoToMeeting app, and the weekly telepractice check-in with the SLP were likely essential aspects of these individuals' success with this technology. 
Table 4 Home Practice (Treatment) Effect: Measures Comparing Postpractice to Posttreatment Phase

\begin{tabular}{|c|c|c|c|c|c|c|}
\hline $\begin{array}{l}\text { Participant/ } \\
\text { TX-HP } \\
\text { Condition }\end{array}$ & $\begin{array}{l}\text { Treatment } \\
\text { (HP) Mean > } \\
\text { Baseline }\end{array}$ & $\begin{array}{l}\text { Visual Inspection } \\
\text { Overlapping } \\
\text { Data Points }\end{array}$ & $\begin{array}{l}\text { Treatment } \\
\text { Data Points }> \\
\text { Baseline Trend Line }\end{array}$ & $\begin{array}{l}\text { \% Change } \\
\text { Relative to } \\
\text { Baseline } \\
\text { Performance* }\end{array}$ & $\begin{array}{l}\text { Buskin and } \\
\text { Serlin } d / \\
\text { Effect Size }\end{array}$ & $\begin{array}{l}\text { Integrated } \\
\text { Effect Size }\end{array}$ \\
\hline \multicolumn{7}{|l|}{$A C L$} \\
\hline TR-PR & Yes & Overlapping & Yes & 133 & $1.44 /$ none & Moderate \\
\hline UNTR-PR & Yes & None & Yes & 1633 & 13.28/large & Strong \\
\hline \multicolumn{7}{|l|}{ MCR } \\
\hline TR-PR & Yes & Overlapping & Yes & 115 & 2.0/none & Strong \\
\hline UNTR-PR & Yes & None & Yes & 227 & 8.27/medium & Strong \\
\hline \multicolumn{7}{|c|}{ NWS } \\
\hline TR-PR & Yes & Overlapping & Yes & 116 & $2.17 /$ none & Moderate \\
\hline UNTR-PR & Yes & None & Yes & 153 & $4.17 / \mathrm{small}$ & Strong \\
\hline \multicolumn{7}{|c|}{ PBS } \\
\hline TR-PR & Yes & Overlapping & No & 116 & 0.83/none & Weak \\
\hline UNTR-PR & Yes & None & Yes & 153 & 3.91/small & Strong \\
\hline \multicolumn{7}{|c|}{ SSM } \\
\hline TR-PR & Yes & Overlapping & Yes & 115 & 4.04/small & Strong \\
\hline UNTR-PR & Yes & None & Yes & 126 & 6.35/medium & Strong \\
\hline
\end{tabular}

Abbreviations: HP, home practice; TR-PR, trained to be practiced; Tx, treatment; UNTR-PR, untrained to be practiced. *Percent change over "baseline" is change from HP versus performance posttreatment: i.e., (mean HP $\times 100$ )/ (mean post-TX).

'Buskin and Serlin, a variation on Cohen's $d$ statistic as cited in Beeson and Robey ${ }^{23,24}$; treatment effect sizes of small (4.0), medium (7.0), or large (10.1) per Robey and Beeson. ${ }^{23,24}$

${ }^{\ddagger}$ Integrated effect size per Ramsberger and Marie, ${ }^{6}$ where "strong" indicates all measures are significant.

Another consideration in discussion of HP technology for persons with aphasia is candidate criteria. Of our eight participants who underwent intensive speech therapy, one participant had matching list errors in his iBooks program, compromising the first 2 months of data. Through no fault of his, and despite his dedication to the HP program, his outcomes regarding trained/untrained and practiced/unpracticed were less interpretable. Additionally, two participants were unable to complete the HP program. Of these, one could not reliably be trained to initiate and correctly use the iPad; moreover, she never completely agreed to the usefulness of "overlearning" what she perceived she had already gained during treatment. The other had personal issues that made the HP seem unimportant at the time. In a sense, neither of these two participants "bought in" to the idea of daily practice on the iPad. Nonetheless, seven of eight participants had never used an iPad prior to being trained for this study, yet many of them learned quite rapidly how to do so. Moreover, success using the iPad was not dependent on intact reading skills. In summary, it appears that motivation to use the technology and adequate training are more important factors than age, aphasia type or severity, or prior experience with computers.

Participants on average reported satisfaction with learning and using the iPad. In particular, they seemed to enjoy the autonomy afforded them to choose when, and for how long, they would practice whichever words they felt like practicing. Moreover, being able to practice from home, as opposed to traveling to a clinic, and being able to check in regularly via telepractice-again, without leaving homewere also advantageous. In the long run, as our ability to harness this technology for delivery of service is fine-tuned, there is reason to be hopeful that the combination of mobile apps, video teleconferencing, social media, and other mobile technology may represent a significant breakthrough in the cost benefit of rehabilitation for chronic aphasia. 
In the current study, participants who lived an hour away and had no independent means of traveling to the clinic were particularly grateful for the autonomy afforded by the iPads. Although there are clear benefits in having such autonomy, it must also be noted that there were no controls for training and overlearning sets of words either for a distinct amount of time or to a set criterion. When (some) participants expressed boredom with practicing the same set of words, they were reminded of the neuroscience-based benefits of massed practice and overlearning. ${ }^{12,17}$ They were also aware of, and encouraged by, the progress they were making on monthly probes. Nonetheless, there is inevitably a trade-off between the autonomy and massed practice enabled by the iPad-delivered HP and the potential for unsupervised practicing of erroneous productions. It will be some time before the community of researchers tackling the problem of automatic speech recognition-or better yet natural language processing-can perfect a product to the point of recognizing aphasic speech and language. Until then, the harnessing of mobile technology to assist and/or treat persons with aphasia is, unfortunately, stuck with this trade-off.

Another potential barrier to successfully integrating tablet-based technology into aphasia rehabilitation concerns issues of disabilityrelated accessibility. ${ }^{4}$ Creating our own HP programs allowed us to control for issues such as aphasia-friendly text (large print, simple language, key words capitalized and/or in bold, etc.), consistency in tasks, and visual simplicity. ${ }^{18}$ Nonetheless, we were limited by inflexible timing and size of interactive icons built into the iBooks software with regard to navigating between pages, chapters, and books. For several of the participants, this was a source of frustration until they achieved a fast enough response for tapping the appropriate icons. Such problems will undoubtedly be alleviated as more apps are developed. For example, when we chose iBooks Author about a year and a half ago, it seemed to be the best, most user-friendly app that could handle video. The most recent version of Pictello (AssistiveWare, Amsterdam, the Netherlands) can now include videos, and may be a better choice for creating "talking books" for some individuals with aphasia. Im- portantly, as more apps are developed and tested for use with persons with aphasia, there will be more opportunities for overcoming such disability-related accessibility issues.

As participants gained expertise in navigating the iBooks and improved their ability to recall practiced words on demand, another source of frustration - and generally a limiting factor of the iBooks app-was its inability to increase task demand. Some of the more mildly aphasic participants quickly grew bored with the tasks and wanted more/other kinds of practice. After the first 2 to 3 months, and depending upon their individual level of proficiency, the SLP gave them a notebook in which to practice more challenging exercises, such as writing the words from memory, writing synonyms for the words, writing sentences that included the words, and so on. If a participant was engaged in such activities, the SLP checked this work during the weekly telepractice meeting and requested that the notebook, along with the iPad and practice log, be brought to the monthly probe meeting. This permitted the SLP to determine if the additional practice was facilitating or interfering with word learning. What we learned from this experience was that a greater variety and more challenging tasks should be built in to the iBooks app, at least for some of the participants.

Similarly, at least one participant was very eager to utilize the iPad for other purposes beyond practicing treated and untreated words. She requested and we set up an e-mail account and electronic banking on the iPad; moreover, she was thrilled to learn about Pandora and became a regular user of all three apps. It is noteworthy that this participant reported considerably less daily practice with the iBooks app than did the other participants. This fact did not appear to interfere with her progress on monthly or post-HP probes. However, it will be interesting to see how the patterns of practice intensity correlate with retention of words across participants on the upcoming maintenance probes. Because one advantage of the HP program is the ability for persons with aphasia to perform unsupervised massed practice, we would predict better outcomes from those who practiced intensely. ${ }^{19-21}$ 
Given that tablets by their very nature afford the user a wide range of choices in entertainment, business, education, relaxation, social communication, and so on-way above and beyond the tools for practicing speech and language that SLPs and aphasia researchers may have in mind-it will become increasingly difficult to control for the influence of other sources of language stimulation in treatment studies that utilize this technology. It is hard not to see that as a blessing, regardless of the loss of partial experimental control.

As tablets are becoming normalized as treatment tools, it is all the more important to ensure the efficacy of so-called "aphasia apps" in treating the symptoms and overcoming the obstacles of aphasia. The current study, along with others in this special issue, are meant to contribute toward forging a path in creating an evidence base for independent HP using tablet devices. As clinicians and researchers move forward in embracing and shaping mobile technology, further research will be required on best practices for training both clinicians and persons with aphasia to maximize individuals' success by capitalizing on their current skills and motivation, and the skills they hope to gain. Furthermore, it may be up to clinical researchers, families, and clinicians working with individuals with aphasia to advocate for so-called "digital curbcuts," 22 the digital equivalent of removing sidewalk curbs to increase mobility and access for those in wheelchairs, to increase the likelihood of making this technology not only accessible to our clients with aphasia, but also relevant to their needs.

\section{ACKNOWLEDGMENT}

This research was supported by funding from the National Institute on Deafness and Other Communication Disorders of the National Institutes of Health under award number R01DC011526 (Kurland, PI). The content is solely the responsibility of the authors and does not necessarily represent the official views of the National Institutes of Health. The authors would also like to thank Kimberly Delude for her assistance in assembling the iBooks.

\section{REFERENCES}

1. Hirsch Atticks A. Therapy session 2.0: from static to dynamic with the iPad. ASHA Perspectives on Gerontology 2012;17:84-93

2. Holland AL, Weinberg P, Dittelman J. How to use apps clinically in the treatment of aphasia. Semin Speech Lang 2012;33(3):223-233

3. Kiran S, Des Roches C, Balachandran I, Ascenso E. Validation of an iPad based therapy for language and cognitive rehabilitation in individuals with brain damage. Poster presented at: Clinical Aphasiology Conference; May 2013; Tucson, AZ

4. Brandenburg C, Worrall L, Rodriguez AD, Copland D. Mobile computing technology and aphasia: an integrated review of accessibility and potential uses. Aphasiology 2013;27:444-461

5. Fink RB, Brecher A, Schwartz MF. A computerimplemented protocol for treatment of naming disorders: evaluation of a clinician-guided and partially self-guided instruction. Aphasiology 2002;16: 1061-1086

6. Ramsberger G, Marie B. Self-administered cued naming therapy: a single-participant investigation of a computer-based therapy program replicated in four cases. Am J Speech Lang Pathol 2007;16(4): 343-358

7. Pedersen PM, Vinter K, Olsen TS. Improvement of oral naming by unsupervised computerized rehabilitation. Aphasiology 2001;15(2):151169

8. Palmer R, Enderby P, Cooper CC, et al. Computer therapy compared with usual care for people with long-standing aphasia poststroke: a pilot randomized controlled trial. Stroke 2012;43(7): 1904-1911

9. Snodgrass JG, Vanderwart M. A standardized set of 260 pictures: norms for name agreement, image agreement, familiarity, and visual complexity. J Exp Psychol Hum Learn 1980;6(2):174-215

10. Masterson J, Druks J. Description of a set of 164 nouns and 102 verbs matched for printed word frequency, familiarity and age-of-acquisition. J Neurol 1998;11:331-354

11. Pulvermüller F, Neininger B, Elbert T, et al. Constraint-induced therapy of chronic aphasia after stroke. Stroke 2001;32(7):1621-1626

12. Pulvermüller F, Berthier ML. Aphasia therapy on a neuroscience basis. Aphasiology 2008;22(6): 563-599

13. Davis GA, Wilcox MJ. Adult Aphasia Rehabilitation: Applied Pragmatics. San Diego, CA: College Hill Press; 1985

14. Carlomagno S. Pragmatic Approaches to Aphasia Therapy. London, UK: Whurr Publishers; 1994

15. Goodglass H, Kaplan E, Barresi B. The Assessment of Aphasia and Related Disorders. 3rd ed. Austin, TX: Pro-Ed; 2001 
16. Kaplan E, Goodglass H, Weintraub S. Boston Naming Test. 2nd ed. Austin, TX: Pro-Ed; 2001

17. Kleim JA, Jones TA. Principles of experiencedependent neural plasticity: implications for rehabilitation after brain damage. J Speech Lang Hear Res 2008;51(1):S225-S239

18. Singh S. Designing intelligent interfaces for users with memory and language limitations. Aphasiology 2000;14:157-177

19. Basso A, Caporali A. Aphasia therapy or the importance of being earnest. Aphasiology 2001; 15(4):307-332

20. Bhogal SK, Teasell R, Speechley M. Intensity of aphasia therapy, impact on recovery. Stroke 2003; 34(4):987-993
21. Cherney LR, Patterson JP, Raymer A, Frymark T, Schooling T. Evidence-based systematic review: effects of intensity of treatment and constraintinduced language therapy for individuals with stroke-induced aphasia. J Speech Lang Hear Res 2008;51(5):1282-1299

22. Elman RE. The Internet and aphasia: crossing the digital divide. Aphasiology 2001;15:895-899

23. Beeson PM, Robey RR. Evaluating single-subject treatment research: Lessons learned from the aphasia literature. Neuropsychological Review 2006;16: 161-169

24. Robey R, Beeson PM. Aphasia treatment: Examining the evidence. Presentation at the American Speech-Language-Hearing Association Annual Convention. San Diego, CA; 2005 